\title{
Recent Developments in the Synthesis of Metal-Tipped Semiconductor Nanorods
}

\author{
Sabyasachi Chakrabortty and Yinthai Chan \\ Department of Chemistry, National University of Singapore, \\ Singapore
}

\section{Introduction}

Semiconductor nanocrystals (NCs), commonly known as quantum dots (QDs), have received great attention over the last two decades due to their, unique size and shapedependent optoelectronic properties, as well as their flexible surface chemistry. While efforts to produce colloidal NCs date back to the pioneering work of Rossetti et al at Bell Labs ${ }^{1}$ and Ekimov et al at the Vavilov State Optical Institute ${ }^{2}$ in the early 1980's, the ability to obtain monodisperse spherical and highly crystalline NCs remained largely elusive until the introduction of the hot injection method by Murray et al. in 1993.3 In this method, organometallic precursors of the semiconductor material are rapidly injected into an organic solvent at an elevated temperature under inert conditions. This results in a rapid cooling of the reaction mixture, effectively separating the nucleation and growth phases of the intended semiconductor NCs. Despite the addition of organic surface-capping groups, assynthesized core NCs typically suffer from poor surface passivation and possess surface trap states. These surface trap states result in fast non-radiative relaxation pathways for photogenerated charge carriers, thus leading to reduced fluorescence quantum yields (QYs) typically on the order of $\sim 10-20 \%$. In order to improve the fluorescence efficiency as well as the photostability of semiconductor NCs, growth of an inorganic shell (typically a wider bandgap semiconductor) is generally adopted. One of the earliest and most widely used techniques for the overcoating of semiconductor NCs even today was introduced by Hines et al. in 1996, ${ }^{4}$ where precursors of the semiconductor shell material are added dropwise to a relatively dilute solution of $\mathrm{NC}$ cores at temperatures sufficiently low to prevent homogeneous nucleation of the precursors or Ostwald ripening of the NC cores. Growth of the semiconductor shell can lead to effective surface passivation of the core NC, leading to near unity QYs in the case of CdSe/CdS core-shell NCs, ${ }^{5}$ although QYs in the range of 50$70 \%$ are more common. The synthetic development of various II-VI, IV-VI and III-V colloidal semiconductor NCs have been reported to date, $3,6,7$ and have led to intense research efforts in the study of their fundamental optoelectronic properties as well as their use in applications in areas as diverse as light-emitting diodes (LEDs), ${ }^{8}$ solar cells, ${ }^{9}$ and biological imaging. ${ }^{10}$

While early efforts in the synthetic development of semiconductor NCs focused on minimizing polydispersity in single component spherical particles such as the nowubiquitous CdSe NCs, a more sophisticated understanding of the underlying mechanisms of 
NC growth over the last decade or so oversaw the field evolving quickly towards more complicated structures ranging from highly anisotropic shapes to multi-component particles bearing more than one nanoparticle within the same nanostructure.11,12 Previous reports have suggested that several experimental parameters are important in controlling the size as well as the shape of the nanoparticles, such as the choice of precursors and their respective concentrations, the reaction temperature, and the types of ligands used.13-17 These parameters play a decisive role in the overall morphology and size dispersity of the nanoparticles ultimately synthesized. To date, semiconductor nanoparticles of a wide variety of different shapes have been reported: rods, ${ }^{18}$ tetrapods, ${ }^{19}$ stars $^{20}{ }^{20}$ cubes, ${ }^{21}$ and hyperbranched structures, 22,23 as illustrated in Fig. 1. Owing to the exhibition of strong quantum confinement effects in semiconductor NCs whose dimensions are comparable to their Bohr exciton radius, the shape of such nanostructures is expected to play a significant role in their size-dependent optoelectronic properties. For example, in the case of CdSe nanorods, fluorescence emission is linearly polarized along the long axis of the $\operatorname{rod}^{24}$ while non-radiative Auger relaxation processes are less pronounced as compared to spherical CdSe NCs. Additionally, it has been suggested that branched semiconductor NCs may be more desirable than their spherical counterparts as the active material in solution-processed photovoltaics due to increased percolation pathways for charge transport. ${ }^{25} \mathrm{~A}$ more recent thrust in the field has been the development of hybrid heterostructures in which different functionalities of individual components may be incorporated into the same nanostructure, thus offering a basis for multifunctional NCs. Hybrid metal-semiconductor nanoheterostructures comprising of a metal nanoparticle in intimate contact with a semiconductor NC serve as a good example of such multi-component systems. Such structures either possess the inherent functionalities of the metal and semiconductor nanoparticle, or unique optoelectronic properties that result from close coupling between the two components.

This chapter traces the development of hybrid metal-semiconductor NCs as a current research trend in the field of colloidal semiconductor NCs. Despite the plethora of examples of different-shaped metal-semiconductor nanostructures reported,12,26-28 metal-tipped semiconductor nanorods are perhaps the most intensively studied, and will be the focus of the chapter. Different wet-chemical synthetic strategies to fabricate various colloidal semiconductor nanorods and their growth mechanisms are presented, and methods to deposit metal nanoparticles at specific sites on nanorods are discussed. The possible applications of such metal-tipped nanorod structures are then elaborated on in the context of their unique physicochemical properties. Finally, we present a relatively recent technique in which previously inaccessible metals may be specifically deposited onto the tips of semiconductor nanorods via a photoinduced mechanism.

\section{Colloidal semiconductor nanorods}

An enormous effort has been made in the past decade or so in developing strategies for the synthesis of colloidal semiconductor nanorods in terms of their composition, morphology, size dispersity and surface properties.11,29-32 Exquisite control over these parameters is undoubtedly very important in terms of gaining a good understanding of the fundamental properties in such systems as well as for their purported applications in solution processed photovoltaics, ${ }^{33}$ catalysis, ${ }^{34}$ etc. The colloidal synthesis of semiconductor nanorods via the hot injection method is in many respects similar to that of spherical NCs, where the injection 
of a high concentration of monomers into a solvent at elevated temperatures results in near instantaneous generation of semiconductor nuclei, whereupon the temperature drops significantly and subsequent monomer consumption occurs primarily via the growth of the NCs. Growth into anisotropic structures involves the formation of a preferred crystalline phase in which the adsorbing surfactant molecules specifically bind to individual crystallographic planes ${ }^{35}$ as illustrated by a wurtzite-based nanocrystal structure in Fig. 2 . This in turn exerts a degree of control over the addition of monomers to specific facets on the growing crystal, resulting in anisotropic growth. Thus it is relatively straightforward to visualize how the exclusive addition of monomers to opposite facets of a spherical construct would eventually result in elongated rod-like structures. The mechanism by which such growth occurs will be elaborated in more detail in the next section which describes the different synthetic strategies to make colloidal semiconductor nanorods of various material compositions.

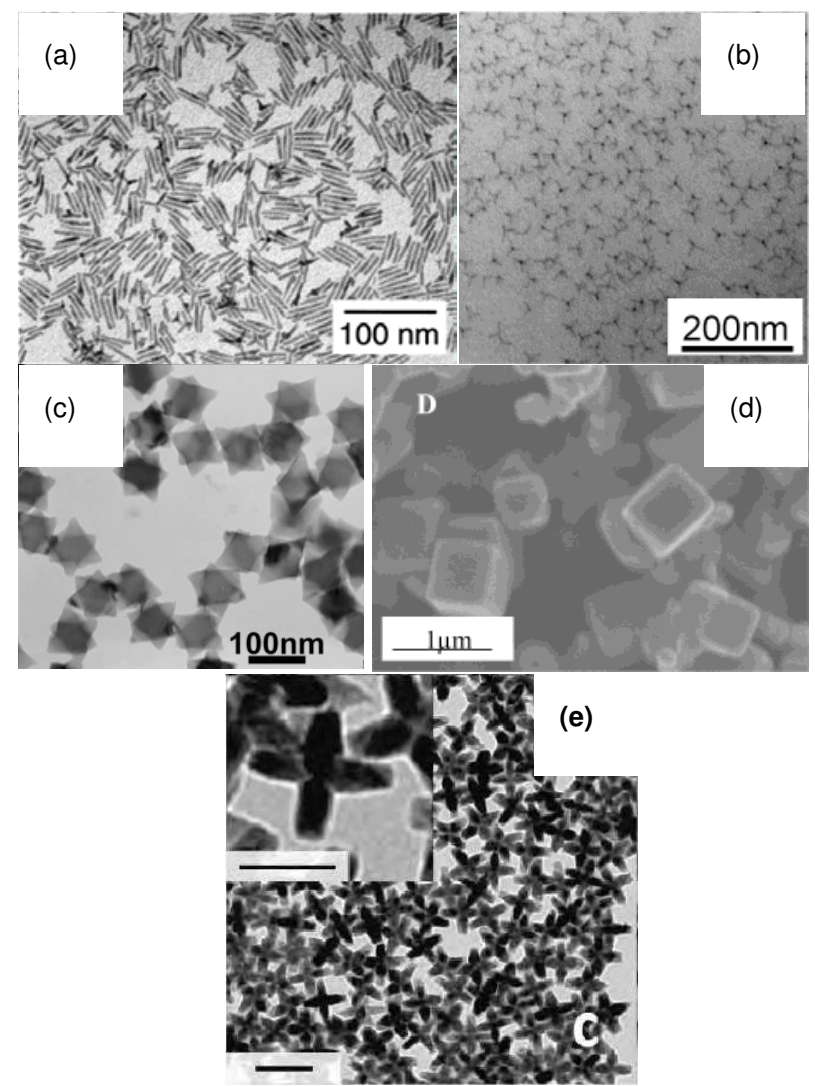

Fig. 1. Examples of colloidal NCs grown with different shapes: TEM images of (a) CdSe nanorods, ${ }^{18}$ (b) CdTe tetrapods, ${ }^{19}$ (c) PbS Stars, ${ }^{20}$ (d) PbSe cubes ${ }^{21}$ and (e) hyperbranched CdSe/CdS octapods (scale bar $100 \mathrm{~nm}$ ). Inset showing one single structure(scale bar $50 \mathrm{~nm}) .23$ Courtesy: (a) J. Am. Chem. Soc. 2000, 122, 12700-12706; (b) Chem. Mater. 2003, 15, 4300-4308; (c) Adv. Mater. 2006, 18, 359; (d) Nano Lett. 2003, 3, 857-862; (e) Nano Lett. 2010, 10, 3770-3776. 


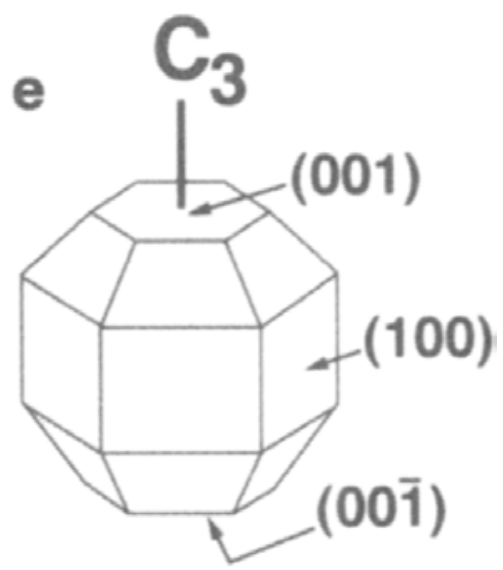

Fig. 2. Model wurtzite nanocrystals showing different facets. Courtesy: J. Phys. Chem. 1995, 99, 17417-17422.

\subsection{Known mechanisms of growth}

\subsubsection{Selective adhesion}

Surfactant molecules which are able to bind to the facets of semiconductor NCs influence their relative growth rates, resulting in anisotropic growth when particular facets are preferred. The extent of growth anisotropy is governed by the binding affinity of the surfactant molecules present in the reaction mixtures to a particular facet, thus influencing the growth kinetics at those facets. It has also been suggested that the anisotropic growth occurs via kinetically driven processes where the system is flooded by a high concentration of monomers. ${ }^{13}$ Under these circumstances, the least passivated facets (and therefore most reactive) will have a tendency to grow faster. In addition to the monomer concentration, the growth times and reaction temperatures are also crucial factors for obtaining rod-like structures of relatively high aspect ratios. Sufficient growth times would be required in order to observe dominant growth long a unique axis while adequately high reaction temperatures facilitate the breakdown of precursors and raise the monomer concentration. In the literature, the anisotropic shape control in binary II-VI semiconductor nanorods are mostly reported on the basis of this selective adhesion technique. Among the II-VI semiconductor nanorods such as $\mathrm{ZnS}, \mathrm{CdTe}$, CdSe and CdS,11,36-38 the latter two are arguably the most well-developed in terms of chemical synthesis. The first wet-chemical synthesis of colloidal semiconductor nanorods (CdSe) via the hot injection method is widely ascribed to Alivisatos, Peng and co-workers in which $\mathrm{Cd}$ and Se precursors were injected into a mixture of n-trioctylphosphine oxide (TOPO) and hexylphosphonic acid (HPA) at $\sim 360^{\circ} \mathrm{C} .{ }^{11}$ Fig. 3 (a) shows different batches of as synthesized CdSe nanorods obtained through this method. The amount of HPA plays a decisive role for obtaining the rod-like structure as it helps to favor growth at the $(00 \overline{1})$ facet of the wurtzite CdSe nanocrystal (see Fig. 2) relative to its other facets. ${ }^{18}$ This results in the anisotropic growth of wurtzite (w-) CdSe nanoparticles along its $\mathrm{C}$-axis into elongated rod-like structures, as exemplified by Fig. 3(b). ${ }^{11}$ Thus with a 
judicious combination of surfactant molecules, growth kinetics at the various facets of semiconductor NCs may be tailored such that semiconductor nanorods of different aspect ratios may be reproducibly synthesized. It should be pointed out that the formation of nanorods in a monosurfactant system cannot be satisfactorily explained using this growth model, however, since it relies heavily upon the distinct binding affinities of different surfactant molecules to the facets of the NC.
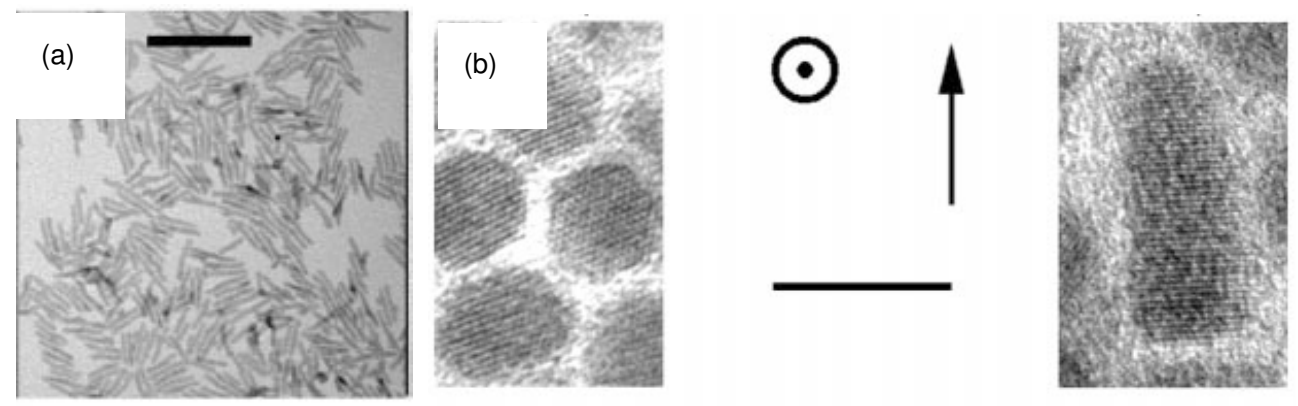

Fig. 3. (a) Low resolution TEM images of colloidal CdSe nanorods (scale bar $50 \mathrm{~nm}$ ). (b) Showing the growth along $\mathrm{C}$-axis to obtain rod shape from spherical nanpparticle(scale bar $10 \mathrm{~nm})$. Courtesy: Nature 2000, 404, 59-61.

\subsubsection{Oriented attachment}

The oriented attachment growth mechanism involves the formation of nearly isotropic spherical NCs and then subsequent fusion of these NCs preferentially along a specific set of crystallographic facets, ultimately leading to the formation of elongated structures. Thus the size dispersity of the resulting rod-like nanostructures is influenced by that of the initial ensemble of spherical particles. Diametrically located regions on the NCs bearing high energy facets serve as nucleation sites for attachment, and the coalescence of the individual crystals along a certain direction may be energetically favorable as it can reduce the existence of the high energy facets, thus providing the driving force for oriented attachment. ${ }^{39}$ The wet-chemical synthesis of colloidal PbSe nanorods with rock-salt structure provides an example of the oriented attachment growth mechanism, where it has been shown that the formation of monodisperse PbSe nanorods, as shown in Fig. 4(a), takes place via the coalescence of spherical PbSe NCs. ${ }^{29}$ Analogous to the synthesis of CdSe nanorods, single crystalline PbSe nanorods may be prepared (Fig. 4) via the introduction of a Se precursor to $\mathrm{a} \mathrm{Pb}$ precursor containing solvent at $\sim 170{ }^{\circ} \mathrm{C}$ in the presence of oleic acid as a surfactant. The presence of high energy $\mathrm{Pb}$ or Se terminated (111) facets in the initial PbSe NC cores were found to be most likely responsible for the oriented attachment into rod-like structures. Use of HRTEM revealed that the synthesized PbSe nanorods are highly crystalline in nature, with no obvious grain boundaries or periodic stacking faults to suggest their formation via the fusion of multiple spherical NCs. In order to prove the oriented attachment mechanism of growth, aliquots at different time intervals were taken from the reaction vessels and carefully characterized via TEM. ${ }^{29}$ The formation of coalesced particles at early reaction times provided strong evidence for the oriented attachment mechanism, as depicted in Fig. 4(c). 


\subsubsection{Seeded growth}

While the previous two mechanisms for nanorod formation described were based on the homogeneous nucleation and growth from the breakdown of semiconductor precursors, another method to fabricate monodisperse colloidal semiconductor nanorods using preformed spherical semiconductor NCs as seeds was reported independently by Carbone et.al. ${ }^{30}$ and Talapin et. al. ${ }^{31,32}$ The resulting nanorod structures thus comprise of a spherical core within a rod-like shell, which in their case were CdSe/CdS core-shell nanorods, or
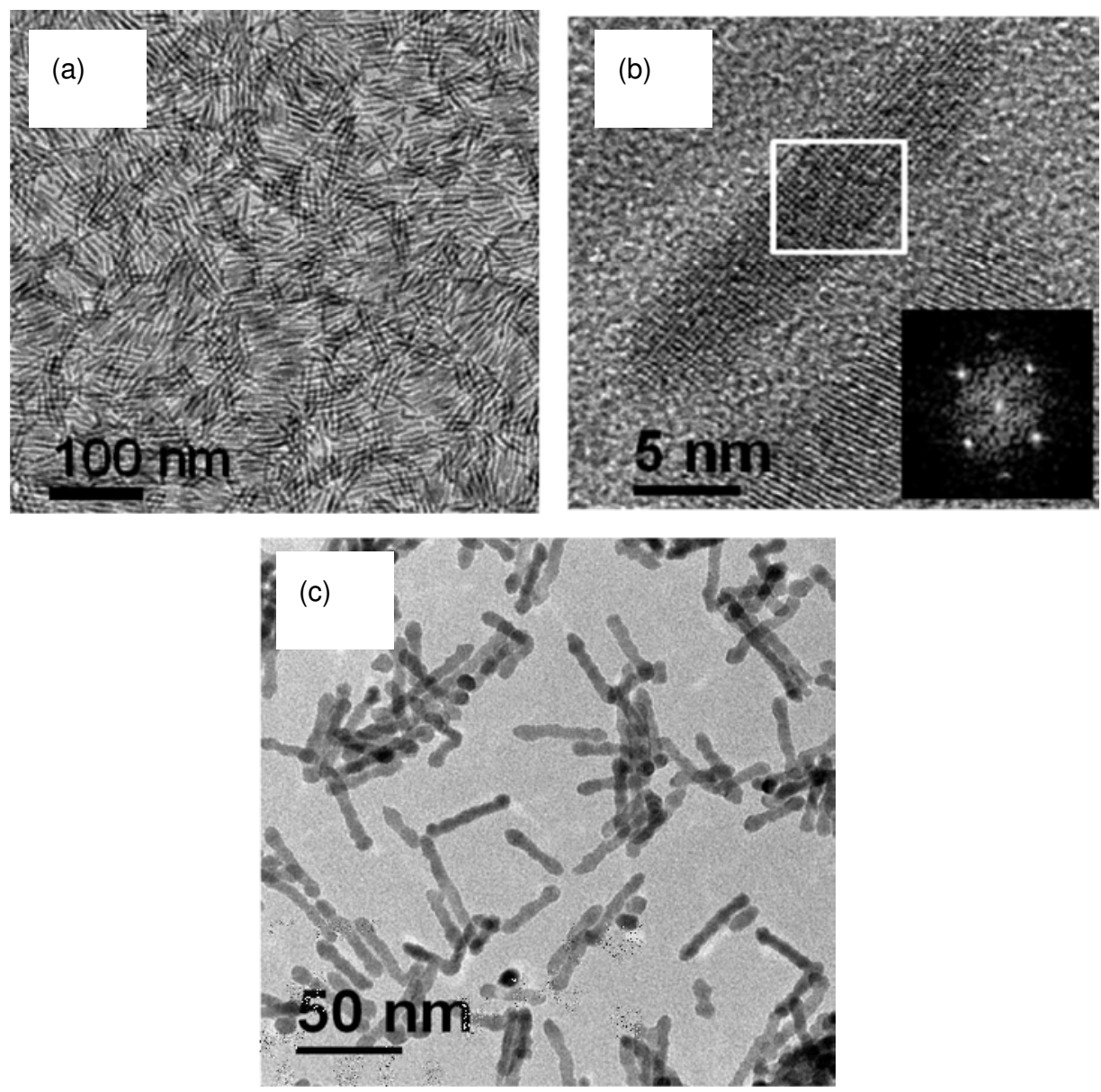

Fig. 4. (a) Low resolution TEM image of PbSe nanorods that formed via an oriented attachment mechanism. (b) It is not readily evident from the High-resolution TEM (HRTEM) image of an individual PbSe nanorod after the synthesis reaction has gone to completion that the formation of the rod occurs through the linear adhesion of a couple of sphere-like PbSe NCs. Inset showing the FFT image. (c) TEM image from an aliquot, taken in the intermediate state of the oriented attachment process. Courtesy: J. Am. Chem. Soc. 2010, 132, 3909-3913. 
perhaps more widely known as CdSe seeded CdS nanorods. For this seeded growth approach, a mixture of spherical CdSe nanocrystal seeds (separately prepared) and a $S$ precursor was injected at elevated temperatures of $\sim 350^{\circ} \mathrm{C}$ to a Cd precursor containing solvent in the presence of alkyl phosphonic acids as surfactants. ${ }^{30}$ As the activation energy barrier for heterogeneous nucleation is generally lower compared with homogeneous nucleation, nearly exclusive heterogeneous nucleation and growth of CdS on the CdSe seed occurs. Owing to the different binding affinities of the alkyl phosphonic acids to the various facets of the CdSe seed and subsequently CdS, continued addition of monomers resulted in a unidirectional growth of the CdS shell, consistent with the growth mechanism described earlier in Section 2.1.1. By obviating the need for homogeneous nucleation, the CdS precursors could be utilized primarily for growth of the shell material on CdSe, thus allowing for very monodisperse rods with a wide range of aspect ratios. Fig. 5 shows transmission electron microscopy (TEM) images of as synthesized CdSe seeded CdS nanorods with aspect ratios ranging from 1:3 to 1:30.30 The CdSe seed was found to be asymmetrically located along the length of the CdS shell due to the fast growth rate along

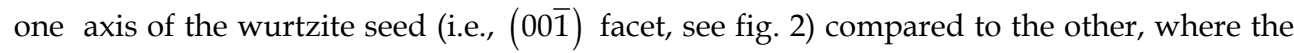
preferential binding affinities of different capping groups play the decisive role. Available examples include $\mathrm{ZnSe} / \mathrm{CdS}, 30 \mathrm{ZnTe} / \mathrm{CdS}, 30 \mathrm{CdSe} / \mathrm{CdTe}^{40}$ etc. Thus, these numerous varieties of materials composition could be further exploited to advance applications in various fields.

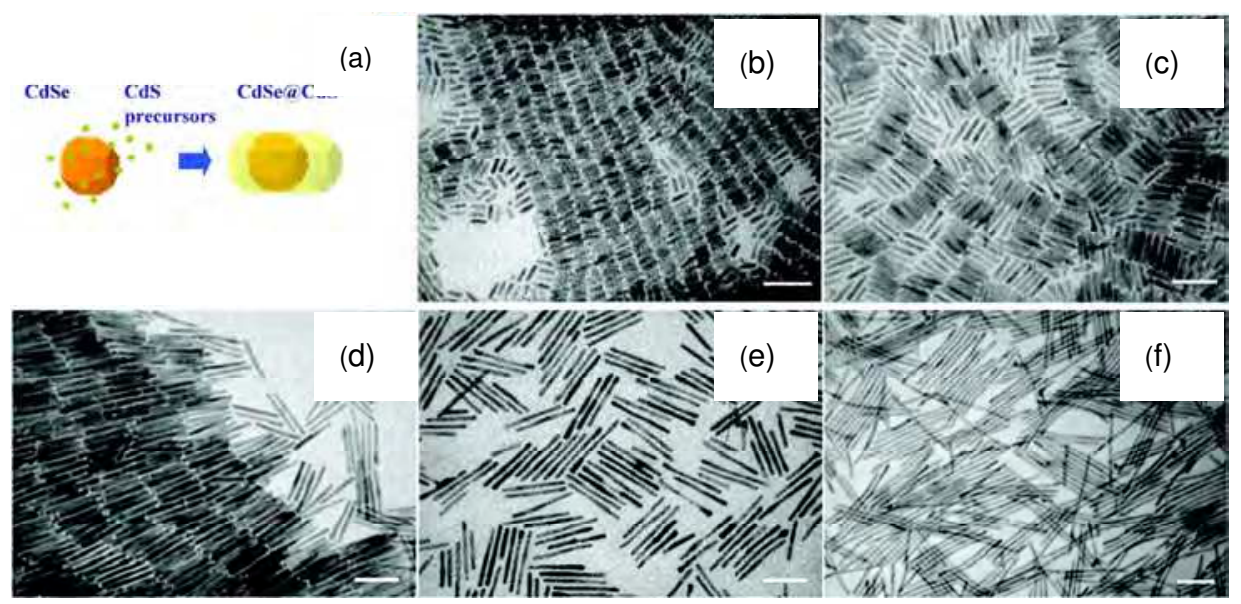

Fig. 5. (a) Schematic illustration of seeded growth approach resulting in the formation of CdSe seeded CdS nanorods. (b) - (f) show CdSe seeded CdS nanorods with aspect ratios ranging from 1:3 to 1:30. Courtesy: Nano Letters 2007, 7, 2942-2950.

\section{Hybrid metal-semiconductor composites}

Multicomponent nanoparticles pave the way to create smart materials by potentially incorporating different functionalities of the individual components within the same nanostructure. Hybrid metal-semiconductor nanostructures are one example of such multicomponent materials, which possibly hold such promise. In these hybrid structures, the metal and its semiconductor nanoparticle counterpart are closely coupled such that 
novel properties or applications may emerge. For example, the selective growth of metal tips onto semiconductor nanorods or tetrapods offers contact points for self-assembly, ${ }^{41}$ good electrical contacts in optoelectronic devices, ${ }^{42}$ modification of its nonlinear optical response ${ }^{43}$ or impart magnetic functionality. ${ }^{44}$ Recently, there has been a fairly large number of examples in the literature where growth of $\mathrm{Pt}^{27} \mathrm{Au}, 26,45$ or $\mathrm{Co}^{28,44}$ onto semiconductor nanorods have been reported.The following section will focus on different synthetic strategies adopted for combining a metal with its semiconductor nanorod counterpart.

\subsection{Synthetic strategy}

It has been shown that the facets at the tips of nanorods such as CdS or CdSe seeded CdS are different in terms of their reactivity when compared with the facets along the length of the rod. The facet reactivities of the two tips of the nanorod themselves differ, and it may generally be concluded that the distribution of facet reactivities on anisotropic semiconductor NCs is quite wide. ${ }^{39}$ The difference in facet reactivities may be exploited to yield selective metal deposition, which may be understood from the standpoint of monomers of the metal precursor exclusively nucleating and growing at the most reactive facets of the semiconductor nanostructure. In terms of thermodynamics, the preferential deposition of a secondary (metal, in this case) particle is governed by minimizing the overall surface and interfacial energy of the system. However, kinetic factors such as reactant diffusion and how labile the surfactant molecules on the semiconductor are play a pivotal role in the fabrication of the hybrid metal-semiconductor nanostructure as well. The next few sections will introduce different techniques that have been reported for the fabrication of hybrid metal-semiconductor nanorods.

\subsubsection{Site selective metal deposition}

It was previously suggested that the atomic arrangements in the apex facets in semiconductor nanorods are dissimilar compared to their longitudinal sidewalls, which gives rise to different surface energies depending on whether it was the facets at the apex or sides of the nanorod that were being considered. This phenomenon is illustrated well by the wurtzite (w-) lattice, which is generally believed to have chemically non-equivalent surface termination at opposing ends of the structure's growth axis. For example, in w-CdS nanorods, one end is $\mathrm{Cd}$ rich whereas the other is $\mathrm{S}$ rich. ${ }^{46}$ As a result, the corresponding reactivity of the facets at the sulfur- and cadmium-rich ends can differ significantly, which would be expected to lead to dissimilar rates of heterogeneous nucleation and growth of metal clusters at the different ends on the nanorod. Saunders et. al., ${ }^{46}$ previously showed that under anaerobic conditions, Au growth occurs preferentially at the S-rich tip of the CdS nanorod. In contrast, dumbbell-like Au-CdS-Au structures are obtained in the presence of $\mathrm{O}_{2}$ (as summarized in Scheme 1) due to etching of the $\mathrm{Cd}$ rich end, which is expected to increase its surface reactivity and allow for $\mathrm{Au}$ nucleation and growth. By taking into account that the reactivities at the sides of the nanorod are very different as compared to its ends, a hierarchical order of free energy barriers to nucleation at the different sites of the nanorod can be anticipated. Under this basis, Chakrabortty et. al. ${ }^{45}$ reported a facile $\mathrm{Au}$ deposition process to synthesize CdSe seeded CdS nanorods of various Au decorated morphologies by using the concentration of the Au precursor as the only adjustable parameter. Fig. 6(a)-(c) summarizes the different morphologies of Au on the CdS rod surface as concentrations of the Au monomers were increased. This trend of selective Au growth 
with increasing concentrations of added Au precursor was reproduced at a variety of fixed temperatures $\left(25-90^{\circ} \mathrm{C}\right.$ ) and sufficiently long growth times (up to $6 \mathrm{~h}$ ), which in turn supports the presence of a hierarchical order of reactivities between the facets at the tips and sides of the nanorod. ${ }^{45}$ In order to exploit this hierarchy of facet reactivities for the fabrication of nanostructures of higher structural complexity and therefore functionality, sequential exposure to precursors of $\mathrm{Au}$ and then $\mathrm{Ag}$ was pursued. Thus the deposition of $\mathrm{Au}$ to give matchstick-like $\mathrm{Au}-\mathrm{CdSe} / \mathrm{CdS}$ nanostructures was followed by exposure to Ag. Unlike the Au precursors, the Ag precursors undergo cationic exchange with CdS, which results in the formation of $\mathrm{Ag}_{2} \mathrm{~S}$ nanoparticles at the end of the nanorod that did not bear a $\mathrm{Au}$ tip. This resulted in novel "Janus-type" dumbbell structures, ${ }^{45}$ where Au is primarily located at one end of the nanorod while $\mathrm{Ag}_{2} \mathrm{~S}$ is located at the other end as shown in Fig. 6(d). Several other groups have also utilized the fact that the differences in facet reactivities at the sides and tips of semiconductor nanorods can be exploited to yield site selective deposition, and a rapidly growing library of metal-tipped semiconductor nanorod heterostructures (e.g. Co-CdSe/CdS28 and Pt-CdSe/CdS27) have been synthesized.
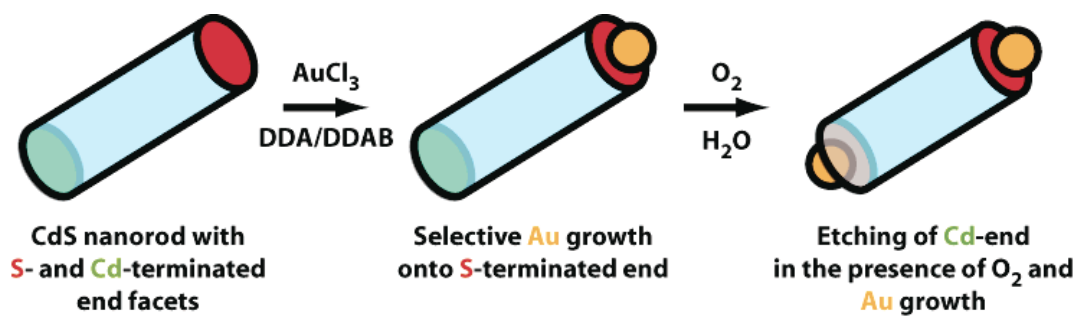

Scheme 1. Schematic of the growth process of Au nanocrystals onto CdS nanorods, where in the absence of $\mathrm{O}_{2} \mathrm{Au}$ deposited on the S-rich facet and in presence Au deposited at both end. . Courtesy: J. Phys. Chem. B 2006, 110, 25421-25429.

\subsubsection{Light controlled metal deposition}

The ground state electronic structure of semiconductors is generally characterized by a valence band that is filled with electrons and a conduction band that is empty. Upon light absorption an electron is promoted from the valence band into the conduction band, leaving behind a hole in the valence band. The photo-generated hole in the valence band and electron the conduction band can react with electron donors and acceptors, respectively, thus allowing the semiconductor surface to act as an oxidation or reduction point. Thus a solution containing metal cations can undergo photoreduction at the semiconductor surface to nucleate and subsequently grow into metal nanoparticles assuming that it is energetically favorable for electron transfer from the semiconductor to the metal to occur. In 2004, Pacholski et. al., 47 demonstrated photoinduced $\mathrm{Ag}^{+}$reduction at the surface of $\mathrm{ZnO}$ nanorods to obtain $\mathrm{Ag}-\mathrm{ZnO}$ nanorod composites, as shown in Fig. 7. The use of stabilizer molecules facilitated the deposition of $\mathrm{Ag}$ monomers primarily at the end of the long $\mathrm{ZnO}$ nanorods, as shown in Fig. 7(b), thus offering a certain degree of control over the site-selectivity of the Ag deposition process. The large, dominant Ag particle growth at a particular region of the nanorod and the absence of smaller clusters of Ag throughout the rod surface strongly suggests that once a small Ag nucleus is formed, the rest of the monomers preferentially add to that nucleus instead of heterogeneously nucleating at other locations on the rod. This photoinduced 
strategy to facilitate the deposition of metals onto a semiconductor surface was not only exploited to synthesize various metal-semiconductor hybrid structures but also to allow for the growth of very large metal domains. As an example, the average diameter of Au domains on $\mathrm{Au}-\mathrm{CdSe} / \mathrm{CdS}$ nanorods synthesized via a thermal growth process ${ }^{48}$ is typically on the order of $\sim 2.0 \mathrm{~nm}$. However, UV irradiation of the Au-CdSe/CdS nanorod causes a transfer of electrons from $\mathrm{CdS}$ to the $\mathrm{Au}$ particle, allowing for the additional reduction of $\mathrm{Au}$ ions in solution at the Au particle surface, thus increasing the size of the Au particle to diameters as large as $\sim 15 \mathrm{~nm}$. ${ }^{8}$ The holes are left behind in the CdS nanorod, and are eventually transferred to the solvent bath. Fig. 8(a) illustrates the growth of large Au domains in Au-CdSe/CdS nanorods via a photoinduced process. Fig. 8(b) is a TEM image showing the dramatic enlargement of the $\mathrm{Au}$ domains on $\mathrm{CdSe} / \mathrm{CdS}$ nanorods via the photoinduced process described above and may becontrasted with the Au-CdSe/CdS structures shown in Fig. 6(a), which were synthesized via a thermal growth process. The addition of hole scavengers such as $\mathrm{EtOH}, \mathrm{CHCl}_{3}$ etc, increase the efficiency of the charge separation by ensuring that there is no buildup of holes in the semiconductor. In summary, this light driven mechanism of getting metals to deposit onto semiconductors may be exploited to achieve more complex hybrid metal-semiconductor nanostructures, as will be elaborated on in a later Section.

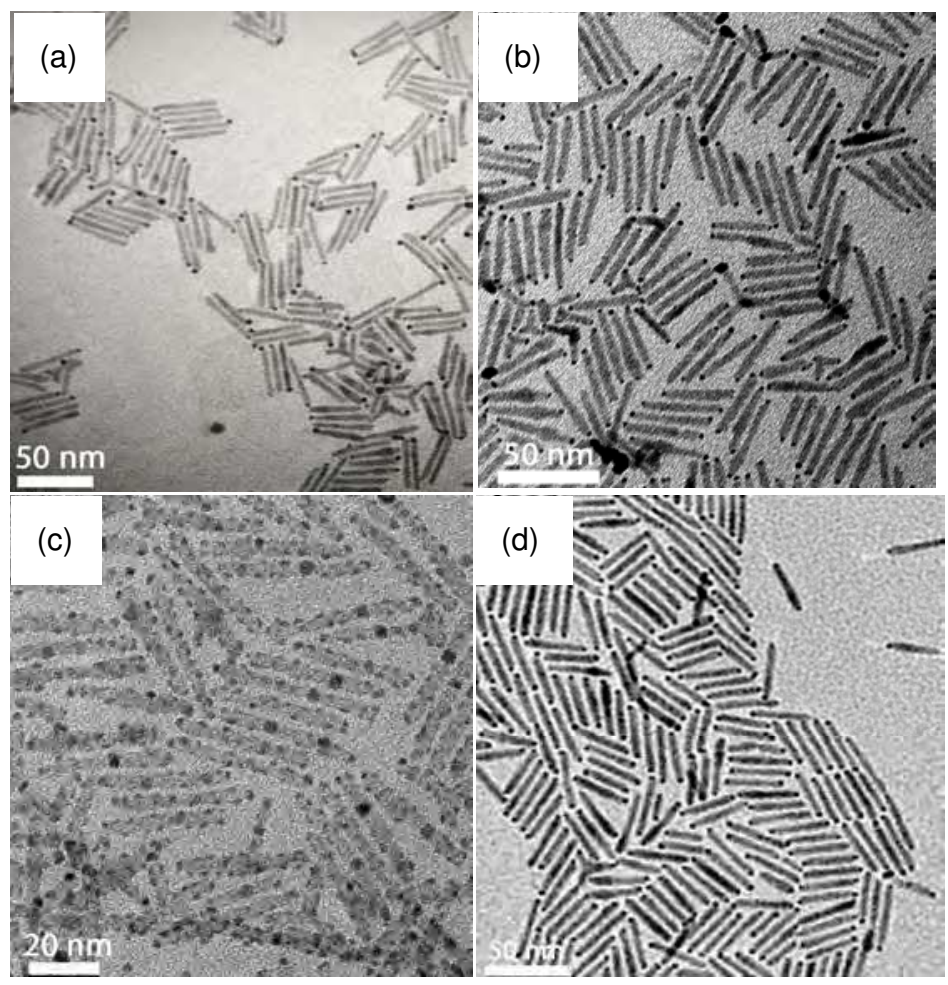

Fig. 6. (a) - (c) are low resolution TEM images showing CdSe seeded CdS nanorods exposed to increasing amounts of Au precursor where the deposition trends proves the hierarchical order of facet reactivities. (d) TEM image of the "Janus-dumbbell" having Au nanoparticle at one end and $\mathrm{Ag}_{2} \mathrm{~S}$ nanoparticle at the other end. Courtesy: Angew. Chem. Int. Ed. 2010, 49, 2888-2892. 


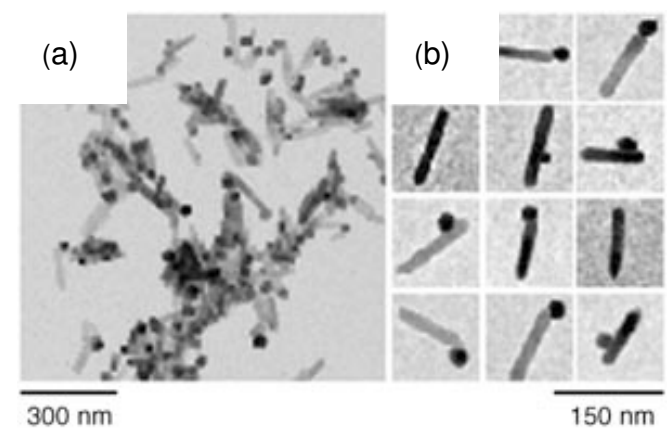

Fig. 7. TEM images of (a) ZnO nanorods with deposited silver particles, (b) gallery of different Ag decorated ZnO surface. Courtesy: Angew. Chem. Int. Ed. 2004, 43, $4774-4777$.

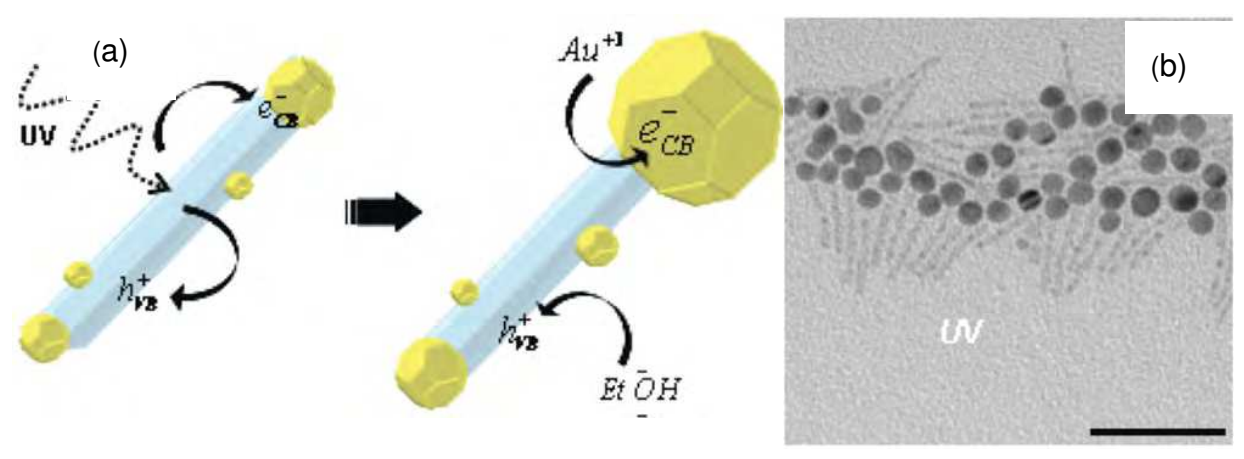

Fig. 8. (a) Schematic, showing the mechanism photo induced large Au growth technique. (b) TEM image of Au-CdS hybrid nanorods formed after UV irradiation having lagre $\mathrm{Au}$ domain (scale bar $50 \mathrm{~nm}$ ). Courtesy: Nano Lett. 2009, 9, 3710-3714.

\subsubsection{Electrochemical Ostwald-ripening}

Ostwald ripening in NCs is a process in which under conditions where all the monomers have been depleted during growth, the smallest and least thermodynamically stable NCs in the ensemble dissolve and re-deposit onto the largest and most thermodynamically stable NCs. While Ostwald ripening in NCs is typically an inter-particle process, it was shown in the case of hybrid Au-CdSe nanorods that the Ostwald ripening of Au occurs via an electrochemical, intra-particle process. While the initial $\mathrm{Au}$ deposition was done via synthetic strategies described in Section 3.1.1, continuation of the reaction after all $\mathrm{Au}$ monomers had been depleted resulted in the transformation of dumbbell-like Au-CdSe to matchstick-like Au-CdSe nanocomposites, ${ }^{26}$ as shown in Fig. 9.

The electrochemical Ostwald ripening process that is relevant to this Au-CdSe nanorod system first involves the dissolution of $\mathrm{Au}$ atoms from the smallest $\mathrm{Au}$ particles into the solution via an oxidative process. The released electrons are shuttled across the semiconductor nanorod surface by hopping through surface states to the largest $\mathrm{Au}$ nanoparticles, where upon reduction of the Au ions in solution occurs. ${ }^{49}$ This entire process is summarized in Scheme 2 below. It is evident from Fig. 9(a) that the two Au domains in 
the dumbbell-like morphology are significantly different in size, and the smaller Au domain possesses a higher surface energy and stronger susceptibility towards oxidation than its larger counterpart, thus providing a driving force for the electrochemical ripening process. The continued dissolution and re-deposition of small Au particles onto the largest ones eventually results in semiconductor nanorods with a single large Au tip.
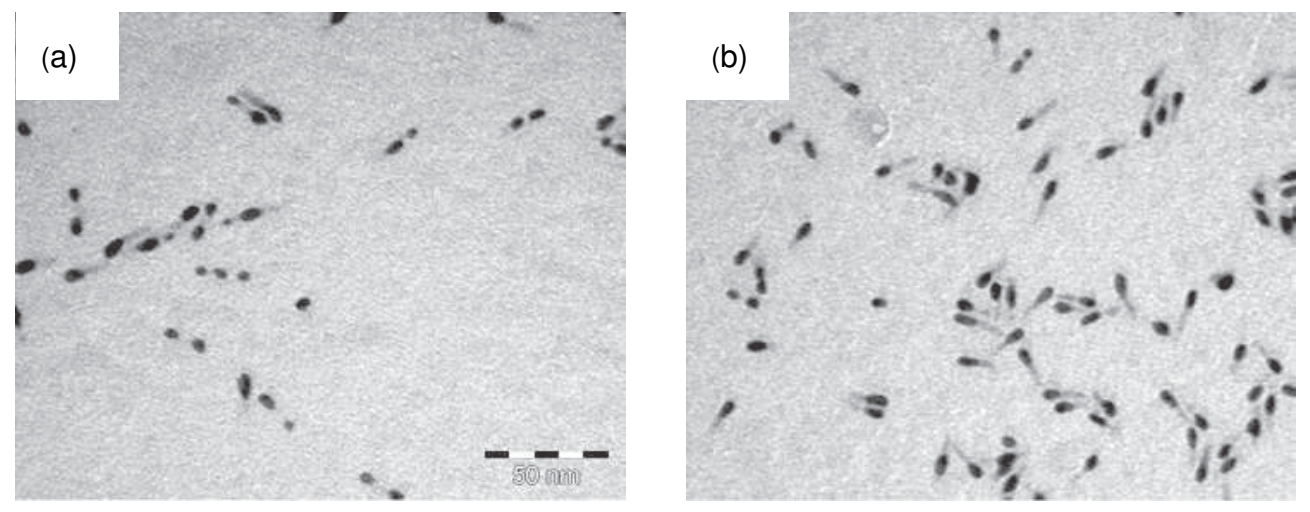

Fig. 9. TEM image of (a) Au-CdSe-Au nanodumbbell and (b) Au-CdSe nano 'nano-belltongues.' Courtesy: Nat. Mater. 2005, 4, 855-863.
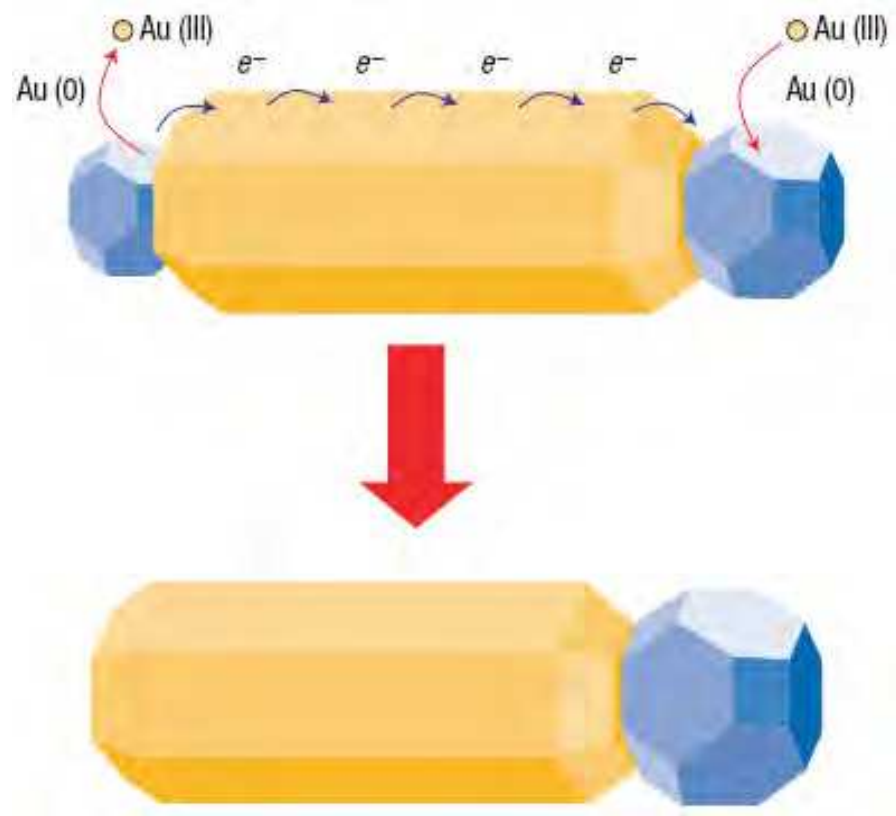

Scheme 2. Schematic of the ripening process in which matchstick-like Au-CdSe nanorods (also known as nano-bell-tongues) are obtained from a dumbbell-like Au-CdSe morphology. Courtesy: Nat. Mater. 2005, 4, 801-802. 


\subsubsection{Surfactant driven metal deposition}

Facet-preferential adhesion of ligands or surfactants may also direct the metal deposition process and the resulting morphology of the hybrid metal-semiconductor nanorod structure. An example of such a system is that of magnetic/semiconductor Co decorated $\mathrm{TiO}_{2}$ nanorods, ${ }^{50}$ where careful variation of the amounts of different surface ligands allowed for the derivation of different Co-decorated $\mathrm{TiO}_{2}$ nanorod morphologies. Through the systematic tuning of different ratios of 1-octanoic acid and oleylamine as strong binding surface capping groups, it was found to be possible to switch from tip-preferential growth to non-selective Co deposition, as summarized in Scheme 3. When the effective concentrations of the ligands are high, they inhibit heterogeneous nucleation on the semiconductor surface, and homogeneous nucleation and growth of Co takes place. A more optimium amount of the surfactants, on the other hand, passivates the sides of the semiconductor surface such that tip selective growth of Co occurs almost exclusively. When exceedingly low amounts of the capping ligands are used, their expectedly insufficient ability to effectively passivate the side facets of the nanorods results in the growth of Co nanoparticles at the tips and throughout the sides of the nanorod. The TEM images corresponding to the various morphologies depicted in Scheme 3 are summarized in Fig. 10. Thus, it is seen from this example that the surfactant can play a very significant role in determining the architecture of anisotropic metal-semiconductor nanostructures.

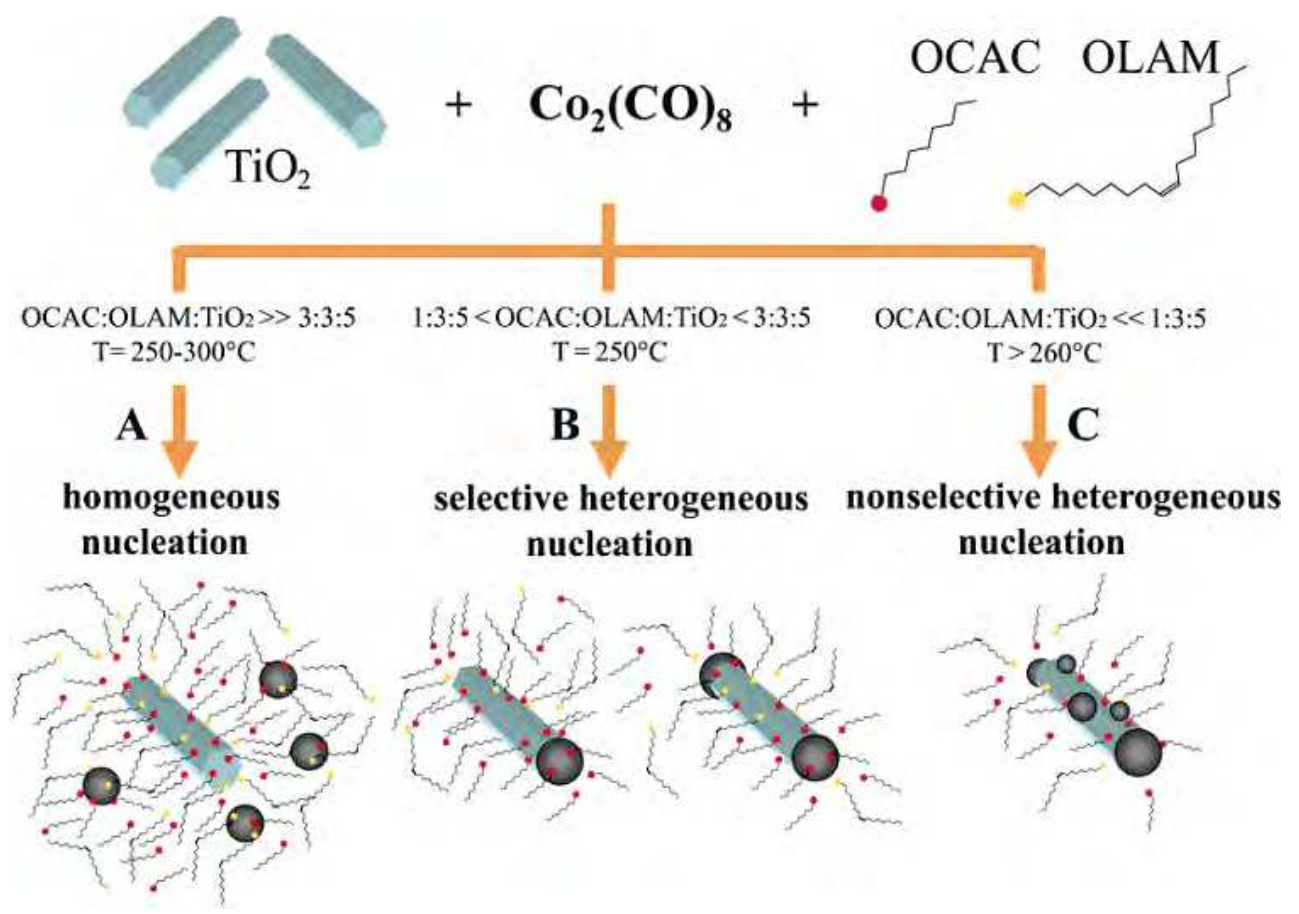

Scheme 3. Schematic for the mechanism of Surfactant-Controlled Co Deposition on $\mathrm{TiO}_{2}$ nanorod Seeds. Courtesy: Nano Lett. 2007, 7, 1386-1395. 


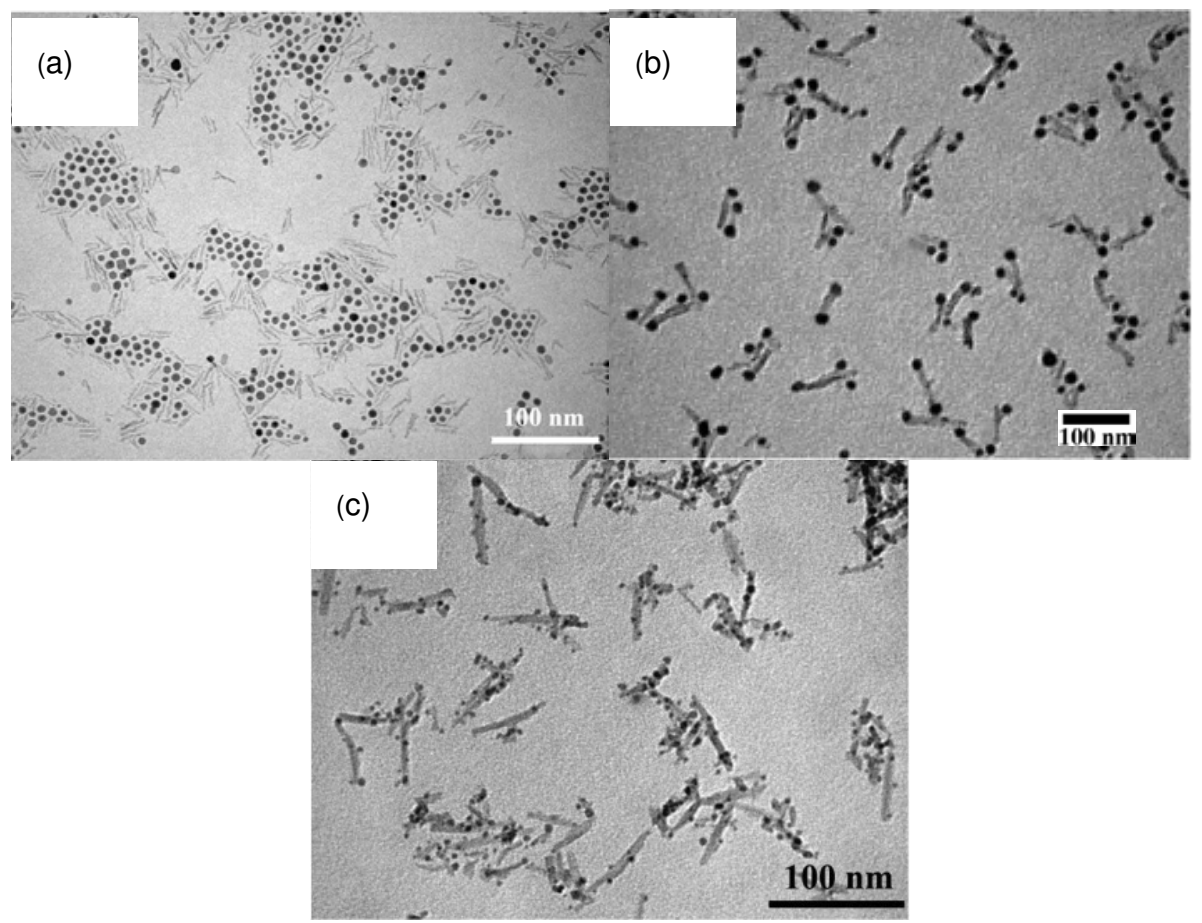

Fig. 10. (a)-(c) Low resolution TEM images corresponding to the various morphologies of $\mathrm{Co} / \mathrm{TiO}_{2}$ hybrid structure, where (a) represents the homogeneous nucleation of $\mathrm{Co}$, (b) shows the tip-preferential growth and (c) illustrate the non-selective Co deposition with $\mathrm{TiO}_{2}$ nanorods. Courtesy: Nano Lett. 2007, 7, 1386-1395.

\subsection{Nature of interface}

As described earlier, various metal nanoparticles can be specifically deposited onto semiconductor nanorods at different locations such as the tip region or throughout the nanorod surface. While the overall morphology of the hybrid metal-semiconductor nanostructure is undoubtedly important from the standpoint of applications in a more general sense, the nature of the interface between the metal and semiconductor plays a pivotal role for charge transfer processes across the metal-semiconductor interface. In the case of Au-Cd chalcogenide nanorods, which is one of the most studied metalsemiconductor nanorod systems, 51,52 it was found that under the mild chemical synthesis conditions reported by most groups, 45 the Au particle typically does not grow epitaxially on the host semiconductor surface, as illustrated in Fig. 11(a). A study by Figuerola et, al.,53 investigated the effects of thermal annealing under solvent-less conditions in vacuum for $\mathrm{Au}$ nanoparticle decorated CdSe nanorods that were deposited onto a substrate, as shown in Fig. 11(b). Upon heating at $250^{\circ} \mathrm{C}$, all small Au clusters along the sides of the nanorods had disappeared, as evident from Fig. 11(c), while it was noticed that the Au domains at the tips grew significantly larger. This phenomenon cannot be ascribed to the intra-particle electrochemical Ostwald ripening described earlier since the dissolution of Au into its ions 
cannot take place without the presence of a solvent. In contrast, it has been proposed that the combination of atomic diffusion and cluster diffusion mechanism is responsible for the steady disappearance of $\mathrm{Au}$ nanocrystals from the lateral facets of CdSe nanorods. These mechanisms can occur on the substrates and were supported by in situ TEM movies.53 Closer investigation of the Au-CdSe region via HRTEM (as shown in Fig. 11(d)) revealed a well-defined epitaxial interface between the Au nanoparticle and CdSe nanorod, thus suggesting that thermal annealing may be a viable strategy towards reconstructing the metal-semiconductor interface in wet-chemically synthesized hybrid metal-semiconductor nanostructures. This would certainly be important in optoelectronic applications involving the transport of charges across the metal-semiconductor interface.

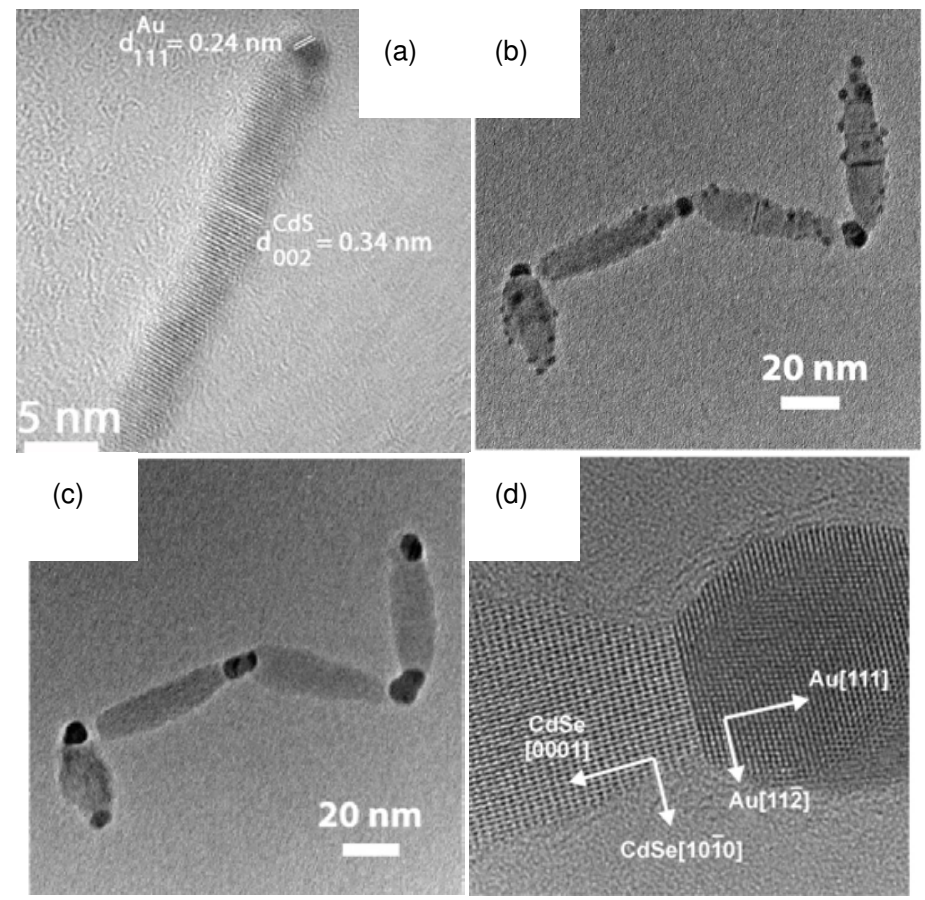

Fig. 11. (a) HRTEM image showing a gold nanoparticle at the apex of the nanorod. (b), (c) TEM images of Au decorated CdSe nanorods heterostructures before and after themmal annealing respectively. (d) HRTEM image of one representative example of epitaxial Au domain with respect to the nanorod. Courtesy: (a) Angew. Chem. Int. Ed. 2010, 49, 2888-2892; (b),(c),(d) Nano Lett. 2010, 10, 3028-3036.

\subsection{Various applications}

As mentioned before, hybrid metal-semiconductor nanostructures can potentially have applications in areas such as directed self-assembly, solution processed optoelectronics and in photocatalysis. Here we describe a few of these applications in order to illustrate the utility of such multicomponent nanostructures. In terms of directed self-assembly processes, the presence of metal tips on semiconductor nanorods provides anchor points for 
functionalizing ligands with functional groups that differ from the ligands bound to the semiconductor. Zhao et. al. fabricated close-packed films of dumbell-like Au-tipped CdSe nanorods ${ }^{54}$ where the rods are aligned parallel to each other due to interactions between alkyl phosphonic acids bound to the CdSe surface, as shown in Fig. 12(b). In addition, the

(a)
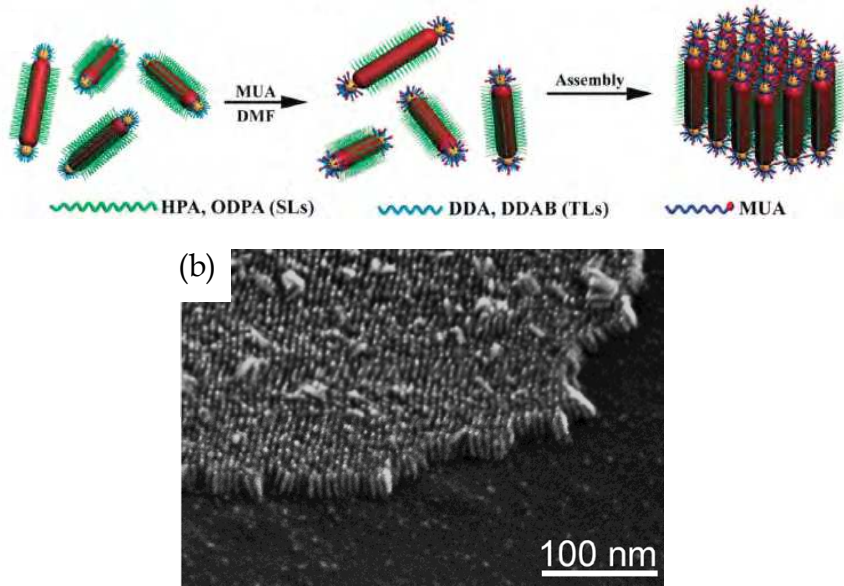

(c)

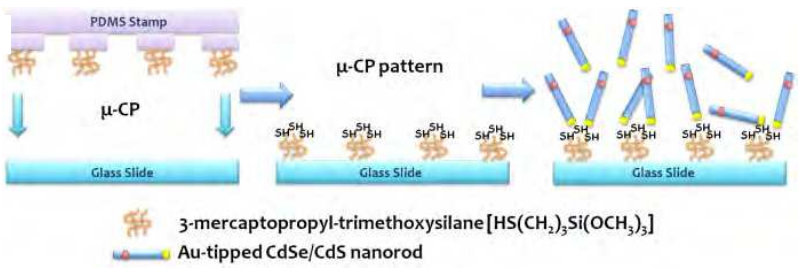

(d)

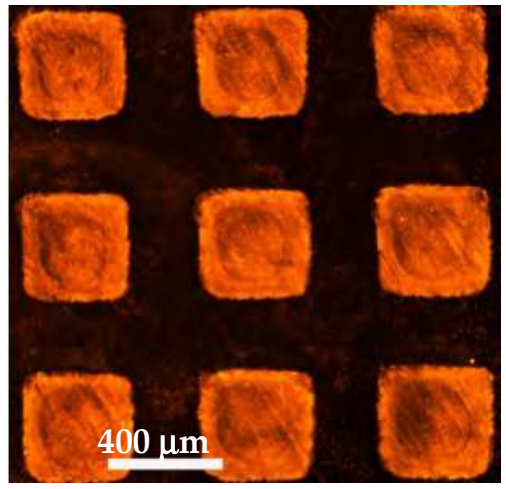

Fig. 12. Examples of the directed assembly of metal-tipped semiconductor nanorods. (a) Schematic illustration of the self-assembly of dumbbell like Au-tipped CdSe NRs (b) SEM image of assembly triggered by interactions between ligands at sides of Au tipped CdSe nanorods. (c) Schematic of directed assembly process with one tipped Au-CdSe/CdS nanorods (d) Showing the fluorescent patterned surfaces on glass substrate. Courtesy: $(a),(b)$ Nano Lett. 2009, 9, 3077-3081; (c),(d) Small 2011, 7, 2847-2852 
formation of closed-packed films were facilitated by the use of 11-mercaptoundecanoic acid which can conjugate with the Au-tips via the thiol moiety and also help to build hydrogen bonding networks through carboxylic acid groups between them. Recently Chakrabortty et. al. demonstrated that careful engineering of the Au-tipped CdSe seeded CdS nanorod architectures can lead to a significant retention of their fluorescence properties,, 5 unlike in the case of Au-CdSe nanorods whose fluorescence are nearly wholly quenched. By patterning a surface with accessible thiol functional groups as illustrated in Fig. 12(c), they were able to show highly specific directed assembly onto the patterned surface via strong Au-S interactions. ${ }^{56}$ Fig. 12(d) is a fluorescence microscope image of Au-tipped CdSe seeded CdS nanorods assembled onto the patterned glass slide. The uniformity of the fluorescence pattern formed showcases the fidelity of the directed assembly process. The photocatalytic properties of metal-semiconductor nanorods was also studied by a number of research groups. 57,58 It was suggested that under light irradiation, charge separation of the photogenerated exciton can take place at the interface between the semiconductor and metal depending on the relative position of the Fermi energy level of the metal with respect to the conduction band of the semiconductor. The transfer of these separated charges to surrounding molecules in the solvent thus becomes a basis for photocatalytic, redox-based reactions. One example of such a system is that of dumbbell-like Au-CdSe, where enhanced charge-separation at the metal-semiconductor interface upon light irradiation leads to the highly efficient degradation of methylene blue (Fig. 13(a)-(b)). ${ }^{57}$ Given the vast combinations of metals and semiconductor nanomaterials that have already been reported, opportunities for exploring the photocatalytic properties of such systems seem numerous. The use of metal tips as electrical contacts for semiconductor nanorods has also been explored at the single nanorod level by Sheldon et. al., ${ }^{42}$ and it was found that the conductance could be enhanced by a factor of at least 100,000-fold compared to standard CdSe rods (Fig. 13(c)-(d)).

\section{Light-induced metal deposition on metal tipped semiconductor nanorods}

Expanding the range of metals that can be deposited onto the semiconductor nanorod surface can result in properties previously inaccessible, opening up avenues for newfound applications or enhancing current ones. However, successful deposition of a particular metal on a semiconductor nanostructure depends on the affinity of the metal and its precursor for the semiconductor. Thus exposure of the CdSe seeded CdS nanorods to Pd precursors under the same reaction conditions as those used for Au deposition does not yield any observable $\mathrm{Pd}$ deposition onto the semiconductor surface, which can be rationalized by the fact that the Pd-S interaction is much weaker than the Au-S interaction on the CdS surface. This can place a limit on the range of metals that can be attached to a given semiconductor. In order to circumvent this problem, Li et. al., 59 reported a deposition approach where existing metal tips in a metal-semiconductor nanostructure are used as heterogeneous nucleation sites for the subsequent deposition of a different metal under conditions of UV photo-irradiation Fig. 14 illustrates the resulting structures when Pd precursors are exposed to Au-tipped CdSe seeded CdS nanorods under UV excitation. It is readily observed that Pd can selectively be deposited onto the Au tipped CdSe seeded CdS nanorods, resulting in a significant increase in particle diameter. Interestingly, it was found from HRTEM (High-Resolution Transmission Electron Microscopy) and HAADF- STEM (high angle annular dark fieldscanning transmission electron microscopy) data that the Pd forms a homogeneous alloy with the Au particle rather than an expected core-shell structure. The successful deposition 
of Pd may be understood as follows: under UV excitation, the photogenerated electron migrates to the Au tip, making it more reducing, while the hole is left behind in the CdS rod and is eventually transferred to the solvent bath by hole scavengers. The $\mathrm{Pd}^{2+}$ ions are then reduced to $\mathrm{Pd}^{0}$ upon coming into contact with the $\mathrm{Au}$ nanoparticle surface, and end up forming an alloy with Au. This strategy was also extended to the deposition of Fe, which can exhibit magnetic properties even upon oxidation.

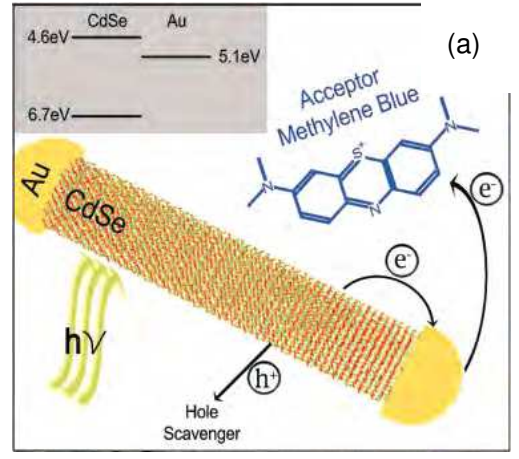

(c)

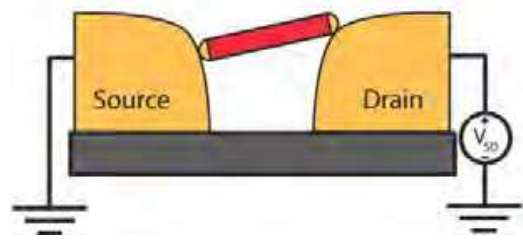

(b)

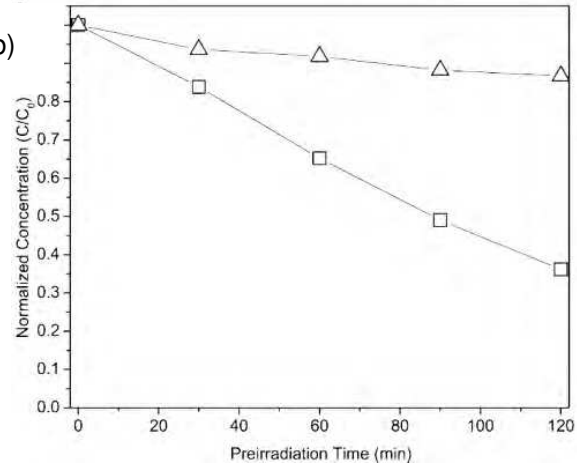

(d)

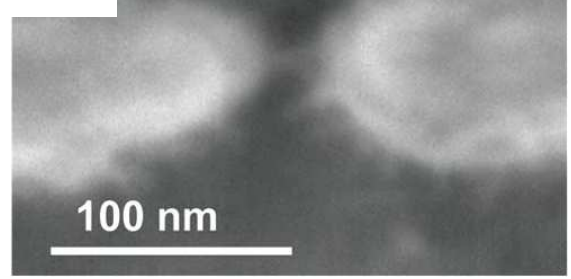

Fig. 13. (a) Scheme of a light-induced charge separation mechanism in Au-tipped CdSe nanorod where the photo-generated electron resides at the gold tip to serve as a reduction point. (b) Comparing the reduction efficiency of CdSe nanorods and gold nanoparticles mixture (blue triangles) with hybrid CdSe-Au nanodumbbells (black squares) in methylene blue dye. (c) and (d) are the schematic and SEM image of a single nanocrystal two-terminal device, respectively. Courtesy: (a), (b) Nano Lett, 2008, 8, 637-641; (c), (d) Nano Lett, 2009, 9, 3676-3682.

Unlike in the case of $\mathrm{Pd}$, however, it was observed that a hollow shell of $\mathrm{Fe}_{3} \mathrm{O}_{4}$ develops around the $\mathrm{Au}$ core at the tip of the CdSe seeded CdS nanorod, as shown by the TEM image in Fig. 15 (a). This was comprehensively verified by HRTEM and HAADF-STEM techniques, as illustrated in Fig. 15(b)-(d). The use of light-induced techniques which exploit the transfer of electrons from the semiconductor to its metal tips in order to facilitate the deposition of other metals that are difficult to grow directly onto the semiconductor surface opens up new avenues for the hierarchical build-up of very complex metal-semiconductor nanostructures. Such structurally complex multicomponent nanoparticles can potentially exhibit unique physicochemical properties that address some of the current outstanding challenges in semiconductor NC-based applications, and perhaps form the basis for new ones. 

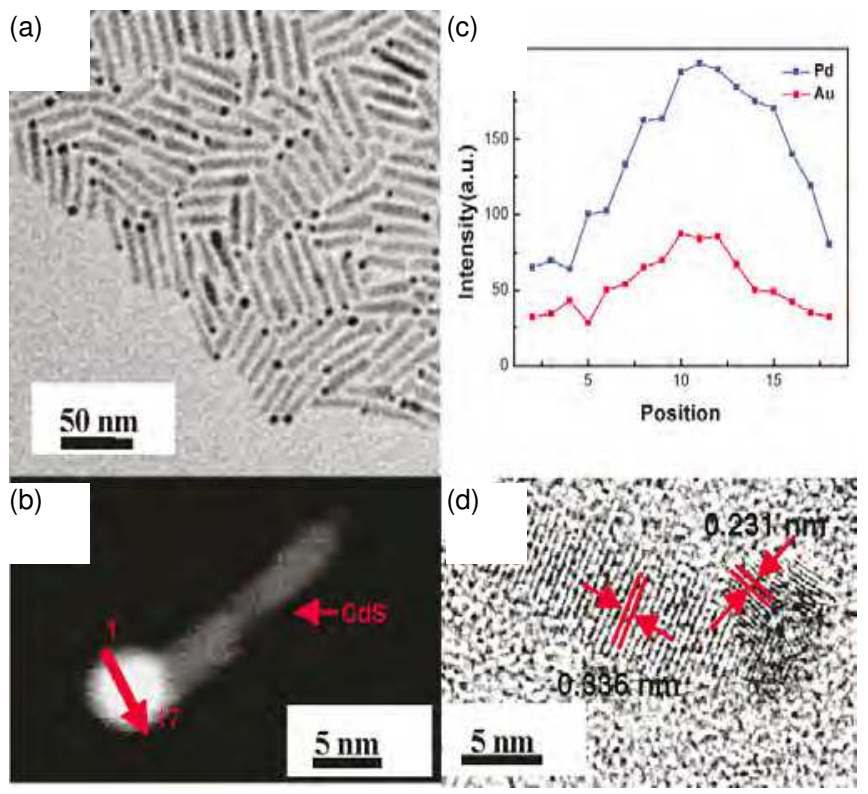

Fig. 14. (a) TEM image of Pd-Au-tipped CdSe-seeded CdS nanorod matchsticklike structures. (b) HAADF-STEM image of a typical rod, showing Au/Pd alloycomposition in it. (c) Corresponding EDX line scan across the spherical tip. (d) HRTEM image of a Pd-AuCdSe-seeded CdS rod. The lattice spacing clearly related to the literature value here. Courtesy: J. Am. Chem. Soc. 2011, 133, 672-675.
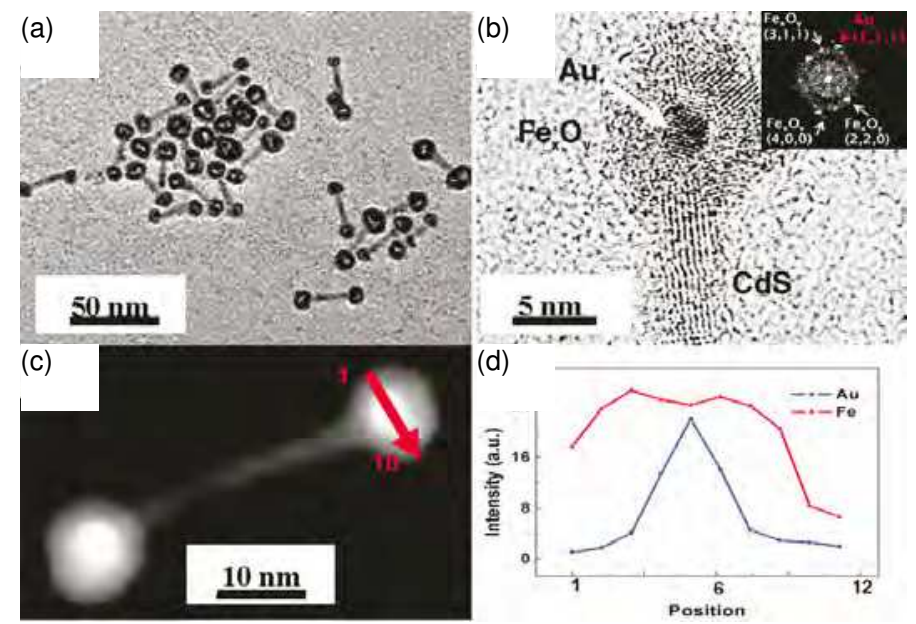

Fig. 15. (a) TEM image core Au nanoparticles with thick hollow shell of $\mathrm{Fe}_{\mathrm{x}} \mathrm{O}_{\mathrm{y}}$. (b) HRTEM image of part of the core-shell-tipped rod. The inset is an FFT image of the spherical tip region. (c) HAADF-STEM image showing three distinct materials composition within the same structure. (d) EDX line scan of the spherical tip shown in (C). Courtesy: J. Am. Chem. Soc. 2011, 133, 672-675. 


\section{Conclusion}

In summary, this chapter describes a diverse set of wet-chemical synthesis routes to obtain monodisperse semiconductor nanorods of different materials composition. One of the most promising synthetic methods for obtaining semiconductor nanorods is that of the seeded growth approach, where very monodisperse rods with aspect ratios as large as 1:30 can be achieved. A number of strategies to achieve a wide range of metal-semiconductor heterostructures was presented, and the conditions used to achieve site-selective deposition were elaborated on. As the nature of the interface between the metal and the semiconductor is of paramount importance in the context of optoelectronic applications, a method to obtain epitaxial growth of metals on semiconductor nanorods was provided. The multicomponent architecture of the hybrid metal-semiconductor nanostructures potentially incorporates multiple functionalities within a single nanoparticle, and can demonstrate utility in a whole host of applications ranging from directed self-assembly to photocatalysis. Some of these salient applications were demonstrated in this chapter, though there is clearly still a lot of progress to be made. Lastly, we described a recently reported light-induced synthetic strategy which potentially expands the range of metals that can be deposited at the tips of semiconductor nanorods. These developments collectively suggest that hybrid metalsemiconductor nanostructures will continue to generate very active research interest from both the standpoints of fundamental science and impactful applications.

\section{Acknowledgment}

We wish to thank the Department of Chemistry, National University of Singapore for its continued support in our research efforts.

\section{References}

[1] R. Rossetti, R. Hull, J. M. Gibson and L. E. Brus, J. Chem. Phys. 1985, 82, 552-559.

[2] A. I. Ekomov and A. A. Onushchenko, JETP Lett. 1982, 34, 345-349.

[3] C. B. Murray, D. J. Norris and M. G. Bawendi, J. Am. Chem. Soc. 1993, 115, 8706-8715.

[4] M. A. Hines and P. Guyot-Sionnest, J. Phys Chem, 1996, 100, 468-471.

[5] H. Han, N. K. Devaraj, J. Lee, S. A. Hilderbrand, R. Weissleder and M. G. Bawendi, J. Am. Chem. Soc. 2010, 132, 7838-7839.

[6] D. Battaglia and X. Peng, Nano Lett. 2002, 2, 1027-1030.

[7] J. M. Pietryga, R. D. Schaller, D. Werder, M. H. Stewart, V. I. Klimov and J. A. Hollingsworth, J. Am. Chem. Soc. 2004, 126, 11752-11753.

[8] S. Coe, W. K. Woo, M. G. Bawendi, and V. Bulovic, Nature 2002, 420, 8706

[9] I. Gur, N. A. Fromer, M. L. Geier, and A. P. Alivisatos, Science 2005, 310, 462

[10] X. Michalet, F. F. Pinaud, L. A. Bentolila, J. M. Tsay, S. Doose, J. J. Li, G. Sundaresan, A. M. Wu, S. S. Gambhir and S. Weiss, Science 2005, 307, 538

[11] X. Peng, L. Manna, W. D. Yang, J. Wickham, E. Scher, A. Kadavanich and A. P. Alivisatos, Nature 2000, 404, 59-61.

[12] T. Mokari, E. Rothenberg, I. Popov, R. Costi and U. Banin, Science 2004, 304, 1787-1790.

[13] Z. A. Peng and X. G. Peng, J. Am. Chem. Soc. 2001, 123, 1389-1395.

[14] M. P. Pileni, Nat. Mater. 2003, 2, 145-150.

[15] S. M. Lee, S. N. Cho and J. Cheon, Adv. Mater. 2003, 15, 441-444. 
[16] C. D. Donega, P. Liljeroth and D. Vanmaekelbergh Small, 2005, 1, 1152-1162.

[17] S. Kumar and T. Nann, Small 2006, 2, 316-329.

[18] L. Manna, E. C. Scher, and A. P. Alivisatos, J. Am. Chem. Soc. 2000, 122, 12700-12706.

[19] W. W. Yu, Y. A. Wang and X. Peng, Chem. Mater. 2003, 15, 4300-4308.

[20] N. Zhao and L. M. Qi, Adv. Mater. 2006, 18, 359

[21] E. Lifshitz,M. Bashouti, V. Kloper, A. Kigel, M. S. Eisen and S. Berger, Nano Lett. 2003, 3, 857-862.

[22] L. Manna, D. J. Milliron, A. Meisel, E. C. Scher, and A. P. Alivisatos, Nat. Mater. 2003, 2, 382-385.

[23] S. Deka, K. Miszta, D. Dorfs, A. Genovese, G. Bertoni and L. Manna, Nano Lett. 2010, 10, 3770-3776.

[24] J. Hu, L. Li, W. Yang, L. Manna, L. Wang, A. P. Alivisatos, Science 2001, 292, 2060-2063.

[25] W. U. Huynh, J. J. Dittmer, A. P. Alivisatos, Science 2002, 295, 2425-2427.

[26] T. Mokari, C. G. Sztrum, A. Salant, E. Rabani and U. Banin, Nat. Mater. 2005, 4, 855-863.

[27] Habas, S. E.; Yang, P.; Mokari, T. J. Am. Chem. Soc. 2008, 130, 3294

[28] Deka, S.; Falqui, A.; Bertoni, G.; Sangregorio, C.; Poneti, G.; Morello, G.; Giorgi, M. D.; Giannini, C.; Cingolani, R.; Manna, L.; Cozzoli, P. D. J. Am. Chem. Soc. 2009, 131, 12817.

[29] W. Koh, A. C. Bartnik, F. W. Wise and C. B. Murray, J. Am. Chem. Soc. 2010, 132, 39093913.

[30] L. Carbone, C. Nobile, M. De Giorgi, F. D. Sala, G. Morello, P. Pompa, M. Hytch, E. Snoeck, A. Fiore, I. R. Franchini, M. Nadasan, A. F. Silvestre, L. Chiodo, S. Kudera, R. Cingolani, R. Krahne and L. Manna, Nano Letters 2007, 7, 2942-2950.

[31] D. V. Talapin, R. Koeppe, S. Gotzinger, A. Kornowski, J. M. Lupton, A. L. Rogach, O. Benson, J. Feldmann and H. Weller, Nano Letters 2003, 3, 1677-1681.

[32] D. V. Talapin, J. H. Nelson, E. V. Shevchenko, S. Aloni, B. Sadtler and A. P. Alivisatos, Nano Letters 2007, 7, 2951-2959.

[33] G. Konstantatos and E. H. Sargent, Nat Nanotechnol, 2010, 5, 391-400.

[34] R. K. Wahi, W. W. Yu, Y. Liu, M. L. Mejia, J. C. Falkner, W. Nolte and V. L. Colvin, J. of Mol. Catal. A: Chemical, 2005, 242, 48-56.

[35] J. J. Shiang, A. V. Kadavanich, R. K. Grubbs and A. P. Alivisatos, J. Phys. Chem. 1995, 99, 17417-17422.

[36] Z. Deng, H. Yan and Y. Liu, Angew. Chem. Int. Ed. 2010, 49, 8695-8698.

[37] I. Gur, N. A. Fromer, M. L. Geier and A. P. Alivisatos, Science 2005, 310, 462-465.

[38] F. Shieh, A. E. Saunders and B. A. Korgel, J. Phys. Chem. B 2005, 109, 8538-8542.

[39] P. D. Cozzoli, T. Pellegrino and L. Manna, Chem. Soc. Rev. 2006, 35, 1195.

[40] P. Peng, D. J. Milliron, S. M. Hughes, J. C. Johnson, A. P. Alivisatos and R. J. Saykally, Nano Letters 2005, 5, 1809-1813.

[41] A. Figuerola, I. R. Franchini, A. Fiore, R. Mastria, A. Falqui, G. Bertoni, S. Bals, G. V. Tendeloo, S. Kudera, R. Cingolani and L. Manna, Adv. Mater. 2009, 21, 550-554.

[42] M. T. Sheldon, P. E. Trudeau, T. Mokari, L. W. Wang and A. P. Alivisatos, Nano Lett, 2009, 9, 3676-3682.

[43] J. Yang, H. I. Elim, Q. Zhang, J. Y. Lee and W. Ji, J. Am. Chem. Soc. 2006, 128, 1192111926.

[44] J. Maynadié, A. Salant, A. Falqui, M. Respaud, E. Shaviv, U. Banin, K. Soulantica and B. Chaudret, Angew. Chem. Int. Ed. 2009, 48, 1814-1817. 
[45] Chakrabortty, S.; Yang, J. A.; Tan, Y. M.; Mishra, Nimai; Chan Y. Angew. Chem. Int. Ed. 2010, 49, 2888-2892.

[46] A. E. Saunders, I. Popov and U. Banin, J. Phys. Chem. B 2006, 110, 25421-25429.

[47] C. Pacholski, A. Kornowski and H. Weller, Angew. Chem. Int. Ed. 2004, 43, 4774 -4777.

[48] L. Carbone, A. Jakab, Y. Khalavka and C. Sonnichsen, Nano Lett. 2009, 9, 3710-3714.

[49] P. D. Cozzoli and L. Manna, Nat. Mater. 2005, 4, 801-802.

[50] M. Casavola, V. Grillo, E. Carlino, C. Giannini, F. Gozzo, E. F. Pinel, M. A. Garcia, L. Manna, R. Cingolani and P. D. Cozzoli, Nano Lett. 2007, 7, 1386-1395.

[51] R. Costi, A. E. Saunders and U. Banin, Angew. Chem. Int. Ed. 2010, 49, 4878.

[52] L. Carbone and P. D. Cozzoli, Nano Today 2011, 5, 449.

[53] A. Figuerola, M. Huis, M. Zanella, A. Genovese, S. Marras, A. Falqui, H. W. Zandbergen, R. Cingolani and L. Manna, Nano Lett. 2010, 10, 3028-3036.

[54] N. Zhao, K. Liu, J. Greener, Z. Nie and E. Kumacheva, Nano Lett. 2009, 9, 3077-3081.

[55] S. Chakrabortty, G. Xing, Y. Xu, N. S.Wee, N. Mishra, T. C. Sum and Y. Chan, Small, 2011, 7, 2847-2852.

[56] D. R. Lide, CRC Handbook of Chemistry and Physics, CRC press, 2000, pp. 9-52.

[57] R. Costi, A. E. Saunders, E. Elmalem, A. Salant and U. Banin, Nano Lett, 2008, 8, 637-641.

[58] E. Elmalem, A. E. Saunders, R. Costi, A. Salant and U. Banin, Adv. Mater. 2008, 20, 43124317.

[59] X. Li, J. Lian, M. Lin and Y. Chan, J. Am. Chem. Soc. 2011, 133, 672-675. 


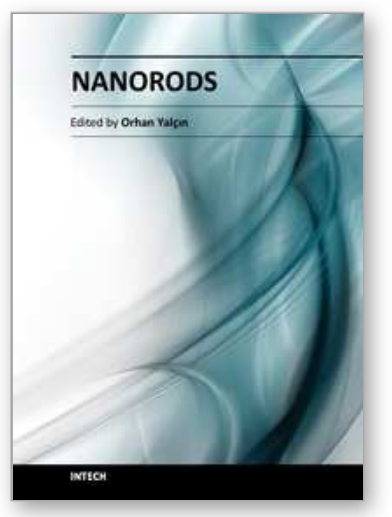

\author{
Nanorods \\ Edited by Dr. Orhan Yalçın
}

ISBN 978-953-51-0209-0

Hard cover, 250 pages

Publisher InTech

Published online 09, March, 2012

Published in print edition March, 2012

The book "Nanorods" is an overview of the fundamentals and applications of nanosciences and nanotechnologies. The methods described in this book are very powerful and have practical applications in the subjects of nanorods. The potential applications of nanorods are very attractive for bio-sensor, magnetoelectronic, plasmonic state, nano-transistor, data storage media, etc. This book is of interest to both fundamental research such as the one conducted in Physics, Chemistry, Biology, Material Science, Medicine etc., and also to practicing scientists, students, researchers in applied material sciences and engineers.

\title{
How to reference
}

In order to correctly reference this scholarly work, feel free to copy and paste the following:

Sabyasachi Chakrabortty and Yinthai Chan (2012). Recent Developments in the Synthesis of Metal-Tipped Semiconductor Nanorods, Nanorods, Dr. Orhan Yalçın (Ed.), ISBN: 978-953-51-0209-0, InTech, Available from: http://www.intechopen.com/books/nanorods/recent-developments-in-the-synthesis-of-metal-tippedsemiconductor-nanorods-

\section{INTECH}

open science / open minds

\section{InTech Europe}

University Campus STeP Ri

Slavka Krautzeka 83/A

51000 Rijeka, Croatia

Phone: +385 (51) 770447

Fax: +385 (51) 686166

www.intechopen.com

\section{InTech China}

Unit 405, Office Block, Hotel Equatorial Shanghai

No.65, Yan An Road (West), Shanghai, 200040, China

中国上海市延安西路65号上海国际贵都大饭店办公楼405单元

Phone: +86-21-62489820

Fax: +86-21-62489821 
(C) 2012 The Author(s). Licensee IntechOpen. This is an open access article distributed under the terms of the Creative Commons Attribution 3.0 License, which permits unrestricted use, distribution, and reproduction in any medium, provided the original work is properly cited. 